\title{
Leptin Fragment Increase Hematopoietic Stem Cell Population and Improve its Engraftment Ability
}

\author{
Carolina C. Dias ${ }^{1}$, Amanda Nogueira-Pedro' ${ }^{1}$, Edgar J. Paredes-Gamero1, and \\ Antonio Miranda ${ }^{2}$ \\ ${ }^{1}$ Department of Biochemistry; ${ }^{2}$ Department of Biophysics, Federal University of São Paulo, \\ São Paulo, SP, 04044-020, Brazil
}

\section{Introduction}

Several studies have shown the important actions of cytokine leptin that regulates food intake and energy expenditure. Additionally, the ability to modulate hematopoiesis has also been demonstrated increasing hematopoietic progenitors. Previous reports of our group have shown that some synthetic sequences of leptin molecules can activate leptin receptor [1-3]. Herein, decapeptides encompassing amino acids from positions 98 to 122 of the leptin molecule were constructed to evaluate their effects on hematopoiesis [4].

\section{Results and Discussion}

All leptin related peptide fragments were synthetized manually by the t-Boc Strategy. Peptides were intraperitoneally administrated in C57BL/6 mice at a dose of $1 \mathrm{mg} / \mathrm{kg}$ for 3 days. After treatment bone marrow cells populations were evaluated. Immunophenotyping was performed by Flow Cytometry (FACSCalibur) in order to quantify hematopoietic stem cells (HSC: $\mathrm{Lin}^{-} \mathrm{c}-\mathrm{Kit}^{+} \mathrm{Sca}-$ $1^{+}$FLK2-Thy $\left.1.1^{\text {low }}\right)$. As observed in Figure 1A the percentage of HSC was increased by the treatment of LEP F. Other peptides (Table 1) did not have effects to alter the percentage of HSC in bone marrow. Additionally, clonogenic assay in methylcellulose was also assessed (Figure 1B).

A

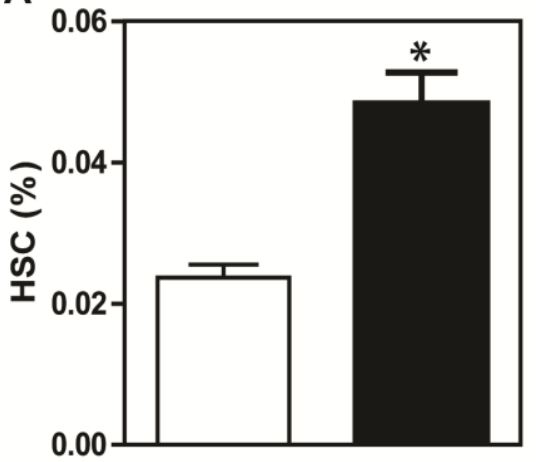

B

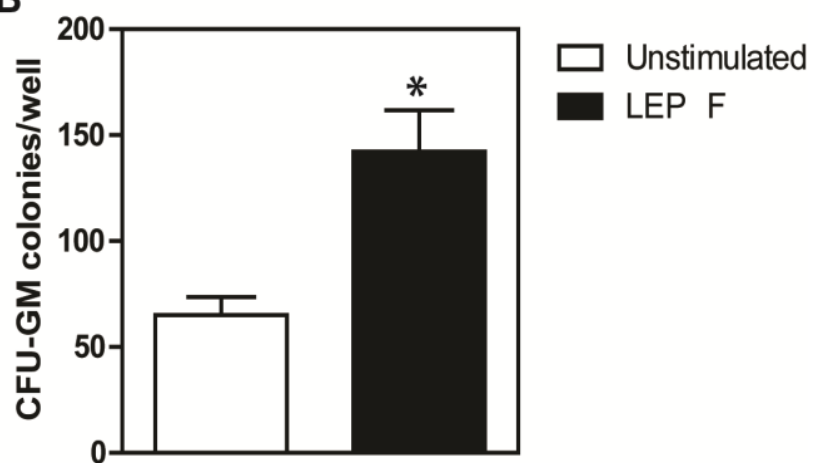

Fig. 1. Effect of LEP F on primitive hematopoietic populations (A) Percentage of HSC (B) The capacity to form primitive $C F U-G M$.

Each colony formed is related with the ability of progenitors to proliferate and differentiate to mature progenies. Treatment of bone marrow cells with LEP F also increase the ability of hematopoietic progenitors to produce colonies. Furthermore, LEP F also improves the grafting of HSC in bone marrow, but did not accelerate the recovery of bone marrow after ablation (data not shown). This results show that this decapeptide have the ability to increase HSC and improve bone marrow transplantation. These features can be helpful in different medical procedures such as bone marrow transplant. The synthetic peptide LEP F was the only peptide that possessed the ability to increase the percentage of hematopoietic stem cells (HSC). Moreover, LEP F also produced an increase of granulocyte/macrophage colony-forming units and activated leptin receptor. Furthermore, LEP F also improves the grafting of HSC in bone marrow, but did not accelerate the recovery of bone 
Table 1. Peptide sequence of the synthetic leptin fragments studied.

\begin{tabular}{|c|c|c|}
\hline Abrev. & Name & Sequence \\
\hline LEP A & Ac-mLep $98-122-\mathrm{NH}_{2}$ & Ac-A-H-D-L-E-N-L-R-D-L-L-H-L-L-A-F-S-K-S-C-S-L-P-Q-T-NH 2 \\
\hline LEP B & Ac-mLep $98-107-\mathrm{NH}_{2}$ & Ac-A-H-D-L-E-N-L-R-D-L-NH 2 \\
\hline LEP C & Ac-mLep $101-110-\mathrm{NH}_{2}$ & Ac-L-E-N-L-R-D-L-L-H-L-NH ${ }_{2}$ \\
\hline LEP D & Ac-mLep $104-113-\mathrm{NH}_{2}$ & Ac-L-R-D-L-L-H-L-L-A-F-NH ${ }_{2}$ \\
\hline LEP E & Ac-mLep $107-116-\mathrm{NH}_{2}$ & Ac-L-L-H-L-L-A-F-S-K-S-NH 2 \\
\hline LEP F & Ac-mLep ${ }_{110-119}-\mathrm{NH}_{2}$ & Ac-L-L-A-F-S-K-S-C-S-L-NH 2 \\
\hline LEP G & Ac-mLep $113-122-\mathrm{NH}_{2}$ & Ac-F-S-K-S-C-S-L-P-Q-T-NH 2 \\
\hline
\end{tabular}

marrow after ablation with 5-fluorouracil. These results show that LEP F is a positive modulator of the in vivo expansion of HSC and could be useful in bone marrow transplantation [5].

\section{Acknowledgments}

This work was supported by grants and fellowships from CNPq and FAPESP.

\section{References}

1. Oliveira, V.X., et al. Regulatory Peptides 127, 123-132 (2005), http://dx.doi.org/10.1016/j.regpep.2004.11.001

2. Martins, M.N.C., et al. Regulatory Peptides 153, 77-72 (2009), http://dx.doi.org/10.1016/j.regpep.2008.11.013

3. Oliveira, V.X., et al. J. Pept. Sci. 14, 617-625 (2008), http://dx.doi.org/10.1002/Pse.957

4. Dias, C.C., et al. Peptides 50, 24-27 (2013), http://dx.doi.org/10.1016/j.peptides.2013.09.012

5. Dias, C.C., et al. J. Cellular Biochemistry (2015), http://dx.doi.org/10.1002/jcb.25090 